\title{
[AB1093] WORSENING OF PAIN AND SWELLING FOLLOWING TRIAMCINOLONE HEXACETONIDE INTRA- ARTICULAR INJECTION: A PROSPECTIVE STUDY IN A COHORT OF PATIENTS WITH RHEUMATIC DISEASES
}

\section{F. Pereira ${ }^{1}$, R. M. dos Santos ${ }^{2}$, R. N. V. Furtado ${ }^{1}$, A. T. de Landa ${ }^{1}$, C. S. P. C. Moleta ${ }^{1}$, P. Bermudes $^{1}$, J. Natour'. ${ }^{1}$ Rheumatology Division; ${ }^{2}$ Unifesp, São Paulo, Brazil}

Background: A worsening articular pain and swelling following triancinolone hexacetonide (TH) intra-articular injection (IAI) is an event understudied.

Objectives: To analyze the frequency and time duration of worsening following intra-articular injection (WFI) in subjects with rheumatic disease; and to determine the association between WFI and demographic or clinical variables.

Methods: A prospective study was carried out in a cohort of patients with rheumatic diseases who had indication of TH IAI. Inclusion criteria: age older than 18 years; refractory synovits caused by either autoimmune or degenerative disorders. Exclusion criteria: uncontrolled comorbidities; and suspected local or systemic infection. Patients received mono-, pauci- or poly- articular TH IAI, according to number of active synovitis (pain and swelling joint). All the IAI was performed blindly and only once. Before the IAI, a questionnaire was administered to the patients, addressing their demographic data; rheumatic disease; comorbidities; medications used and lifestyle habits. An observer kept contact by phone, daily, for eight consecutive days following the injection to identify possible factors associated to WFI. The following questions were asked about: worsening of pain Visual Analog Scale (VAS) and swelling VAS scores after IAI; start time and time duration of WFI. Associations analysis were made. A $5 \%$ level of significance was employed.

Results: Sixty nine patients were included in this study, 63 (91.3\%) were women; 18 (26.1\%) and 39 (56.5\%) had no-white and white skin color, respectively. The average age was $56.4 \pm 14.8$ years; thirty five (50.7\%) had rheumatoid arthritis (RA), and $34(49.3 \%)$ have osteoarthritis; the mean duration of the disease was $9.8 \pm 6.5 y e a r s$. One-hundred and one TH IAI were performed, 28 (40.6\%) were mono-articular injection; 38 (55.1\%) pauciarticular injection; and 3 (4.3\%) poly-articular injection (>4 joints). Regarding the joint, 79 (60.3\%) were small and medium size joints and 52 (29.7\%) were large joints (knee). Fifty-eight (84.1\%) of patients presented worse in pain and swelling after IAI what is consider an WFI and it occurs in 113 joints (86.3\%). Regarding the joints that present WFI 84 (74.3\%) were small and medium joints and 29 (25.7\%) were large joints (knee). The mean time duration of WFI was 17.2 \pm 12.5 hours; and the mean of pain and swelling VAS worsening were $6.7 \pm 2.7$ and $4.4 \pm 3.2$ respectively. There were statistical associations between WFI time duration and following variables: RA diagnosis $(p=0.040)$ and female gender $(p<0.001)$. There were statistical associations between pain VAS worsening and smoke habit $(p<0.001)$; between swelling VAS worsening and variables as: RA $(p=0.023)$, smoke habit $(p=0.031)$ and the small joint injected $(p=0.023)$.

Conclusions: More than $80 \%$ of injected patients related self limited WFI, and this happened mainly in RA patients and smokers.

\section{References:}

Habib GS et al (2010). Local effects of intra-articular corticosteroids. Review article. Clin Rheumatol.

Furtado RNV et al (2005). Polyarticular corticosteroid injection versus systemic administration in treatment of rheumatoid arthritis patients: a randomized controlled study. J Rheumatol.

Disclosure of Interest: None declared

DOI: $10.1136 /$ annrheumdis-2014-eular.3674

Citation: Ann Rheum Dis 2014;73(Suppl2): 1163

Session: Epidemiology, health services and outcome research 